\title{
O PAPEL DO FARMACÊUTICO MAGISTRAL NA PREVENÇÃO DE ESTRIAS GRAVÍDICAS
}

Jaqueline Maisa FRANZEN ${ }^{1}$

Talize FOPPA ${ }^{2}$

Vilmair ZANCANARO

\begin{abstract}
${ }^{1}$ Graduando do curso de Farmácia da Universidade Alto Vale do Rio do Peixe. franzenjaqueline@ gmail.com
${ }^{2}$ Farmacêutica, Mestre em Controle de Qualidade. Coordenadora e Docente do curso de Farmácia da Universidade Alto Vale do Rio do Peixe. talize@uniarp.edu.br

${ }^{3}$ Farmacêutica, Especialista. Mestranda em Ciência e Biotecnologia. Docente do curso de Farmácia da Universidade Alto Vale do Rio do Peixe. Farmacêutica Responsável pela Farmácia Escola de Manipulação da Farmácia da Universidade Alto Vale do Rio do Peixe. vilmair@uniarp.edu.br
\end{abstract}

Recebido em: 24/08/2015 - Aprovado em: 06/01/2016 - Disponibilizado em: 30/07/2016

Resumo: A gravidez é um período onde ocorrem muitas mudanças no organismo da gestantes. Estas mudanças interferem no sistema imunológico, hormonal, vascular que deixam as mulheres mais propensas a alterações cutâneas. As estrias gravídicas oustriae gravidarum são comuns entre as gestantes e costumam aparecer no segundo e terceiro trimestre gestacional, pois é neste período que ocorre o aumento de peso brusco, resultando na distensão da pele formando as estrias gravídicas. A prevenção das estrias leva em consideração diversos fatores que variam desde a hidratação da pele até alimentação adequada. Muitas gestantes não recebem orientações sobre os cuidados com a pele durante o período gestacional, agravando a incidência das estrias. A associação de mais de um ativo na formulação se mostra mais eficaz do que ativos isolados para profilaxia do aparecimento das estrias. A maneira em que esta fórmula magistral será aplicada também interfere no êxito do tratamento, juntamente com orientações básicas. Durante a Atenção Farmacêutica a avaliação e anamnese da paciente são indispensáveis. $O$ farmacêutico magistral deve auxiliar na escolha dos ativos para compor a formulação adequada de acordo com cada caso, orientando a paciente sobre a aplicação da formula e também orientar sobre os fatores que irão contribuir para o sucesso do tratamento preventivo.

Palavras-chave:Gravidez. Estrias Gravídicas. Atenção Farmacêutica. Farmacêutico Magistral

\begin{abstract}
Pregnancy is a period where many changes occur in the body of pregnant women. These changes interfere with the immune, hormonal system, vascular that leave women more prone to skin changes. The gravidic streaks or striaegravidarum are common among pregnant women and usually appear in the second and third trimester, it is in this period that there is an increase of sudden weight, resulting in distension of the skin forming the gravidic streaks. Prevention of stretch marks takes into account several factors ranging from skin hydration to adequate food. Many expectant mothers do not receive guidance on skin care during pregnancy, exacerbating the incidence of stretch marks. The association of more than one active in the formulation shown more effective than isolated active for prophylaxis of the appearance of striae. The way in which this magistral formula will also be applied interferes with successful treatment, along with basic guidelines. During the pharmaceutical care evaluation and patient history are indispensable. The masterful pharmacist must assist in the choice of assets to compose the proper formulation according to each case, guiding the patient on the application of the formula and also guidance on the factors that will contribute to the success of preventive treatment.
\end{abstract}

Keywords: Pregnancy. Striaegravidarum.Masterly pharmacist. 


\section{INTRODUÇÃO}

Durante a gravidez ocorrem alterações imunológicas, metabólicas, hormonais e vasculares (TEIXEIRA et al., 2013), que deixam a pele da mulher mais vulnerável a mudanças fisiológicas (URASAKI, 2011). A gestação é um período de grande variação no eixo hipotálamohipófise-adrenal materno (COSTA et al., 2009).

O desenvolvimento de estrias é muito comum entre as gestantes. A incidência neste grupo pode chegar á 87,7\% (GHASEMI et al., 2007). Contudo, outros fatores como a puberdade, aumento brusco de peso, obesidade grave, uso de glicocorticoides, esteroides eaumento excessivo de massa muscular também podem desencadear a formação de estrias (BORGES, 2010)

As estrias são lesões cutâneas dérmicas que se formam em áreas com intensa distensão da pele (COSTA et al.,2009). Atualmente constituem um desafio para o campo farmacoterapêutico. Apesar de não integrar condição dermatológica preocupante do ponto de vista clínico, desencadeiam efeito impactante na condição estética e em alguns casos até psicológica em mulheres (KEDE; SABATOVICH, 2009).

As estrias podem receber o nome destriae distensae ou estrias de distensão (SD). Quando ocorrem no período gestacional são denominadas striae gravidarum ou estrias gravídicas(SG). As SG aparecem na região do abdômen, seios, nádegas, quadris e coxas (THOMAS; LISTON, 2004) e surgem em média na 27,57 $( \pm 5,38)$ semana de gestação (GHASEMI et al., 2007).

Inicialmente no desenvolvimento das estrias,ocorreo processo inflamatório ea derme pode apresentarestado edematoso (KEDE; SABATOCIVH, 2009) neste caso, são classificadas comoestrias iniciais. Apresentam-seainda de forma atrófica com aspecto de hipocromia, e também nacaradas ou antigas dispondo de tecido fibroso esbranquiçado (FERNANDES, 2012). Quando visualizadas sob microscopia óptica, as estrias exibemfibras elásticas adelgaçadas e em menor número (COSTA et al., 2009). Estas modificações ocorrem pelo rompimento da rede de fibras elásticas da derme, que sofrem alterações estruturais (WATSON et al., 1998).

\section{ORIENTAÇÃO FARMACÊUTICA E PREVENÇÃO DE ESTRIAS GRAVÍDICAS}

A prevalência de estrias em gestantes primíparas varia de 52\% $(\mathrm{n}=309)$ (ATWAL et al, 2006) á 87,7\% ( $\mathrm{n}=118)($ GHASEMI et al., 2007). As SG constituem queixas frequentes entre as gestantes, seguida por cansaço nas pernas e retenção de líquidos (COUTINHO et al. 2012).

$\mathrm{O}$ maior risco de desenvolver estrias está associados à mulheres mais jovens, com 
obesidade maternal, com história familiar positiva de SG, ganho de peso excessivo durante a gestação (DURMAZLAR; ESKIOGLU, 2009; OSMAN et al., 2007; MAIA et al., 2009). Á medida que a faixa etária gestacional aumenta, essa proporção parece decrescer (MAIA et al., 2009). Ghasemiet al. (2007) revelou que $87,7 \%$ das gestantes começaram desenvolver as SG próximo à 27,57 $( \pm 5,38)$ semana de gestação. Além disso, maioria das mães que deram a luz de criança com mais de $3,5 \mathrm{~kg}$ desenvolveram SG no decorrer da gestação (MAIA et al., 2009).

Estudo sobre os cuidados de pele adotados por gestantes revelou que 56\% $(\mathrm{n}=124)$ das gestantes não ter recebem qualquer orientação sobre cuidados serem tomados com a pele na gravidez (URASAKI et al., 2011). É de grande importância identificar e na medida do possível intervir nos fatores etiológicos envolvidos no desenvolvimento das SG e uma anamnese completa se faz necessária (COSTA et al., 2009). O farmacêutico deve atender as dúvidas das gestantes, avaliando o grau de conhecimento necessário e diante o expostoavaliar o grau de compreensão da paciente a ser orientada. Cabe ao farmacêutico dividir a entrevista em cinco partes, apresentação, anamnese farmacológica, análise das informações, desenvolvimento da educação e finalização (MARIN et al., 2003).
Pesquisa com dermatologistas encontrou os motivos pelos quais os mesmos prescrevem produtos manipulados. A prescrição dos produtos é devido ao menor preço $(66,7 \%)$, por terem confiança terapêutica $(71,4 \%)$ e pela qualidade do produto $(95,2 \%)$ (SCHEEREN, 2009) onde a maioria prefere prescrever cremes e loções às gestantes (COUTINHO et al. 2012).

A assistência Farmacêutica deve ser voltada à promoção, proteção e recuperação da saúde individual ou coletiva, que vai o desenvolvimento e a produção de medicamentos e insumos ao acompanhamento e avaliação de sua utilização (BRASIL, 2004a, p.01). Para realizar o processo de intervenção e/ou acompanhamento farmacoterapêutico, o farmacêuticodeve ajudar na resolução dos problemas que se relacionam com a farmacoterapia. Quando necessário,deve-se encaminhar o paciente para o médico ou outros profissionais da saúde, prezando o multiprofissionalismo (MELCHIORS, 2013).

Das orientações repassadas as gestantes, algumas se destacam no contexto de prevenção das SG, como: orientações para manutenção do peso (YOSIPOVITCH; DEVORE; DAWN, 2007; NUSSBAUM, BENEDETTO, 2006), incentivo a manter ou adotar um estilo de vida ativo durante o período gestacional (AMERICAN COLLEGE $\mathrm{OF}$ OBSTETRICIANS AND GYNECOLOGISTS, 2003),hidratação diária 
da pele,desestimulo ao uso excessivo de sabonetes e a temperatura elevada durante o banho, (KEDE, SABATOVICH, 2009).

Quanto á prática de atividade física gestacional, exercícios físicos em intensidade leve a moderada(AMERICAN COLLEGE OF OBSTETRICIANS

AND

GYNECOLOGISTS, 2003) associadoà dieta equilibrada, resultam em ganhos favoráveis para a gestante e feto (NASCIMENTO et al., 2011; NASCIMENTO et al., 2011; MOTTOLA, 2009). Uma vez que o excesso de peso pré-gestacional é comum e três vezes mais frequente que o baixo peso em gestantes (CARVALHAES et al., 2013), faz-se necessário a orientação adequada à paciente.Em conformidade com Urasakiet al. (2011), 86,3\% (n=124) das gestantes não pratica nenhuma atividade física, e apresenta alimentação inadequada, além de baixo consumo hídrico.

Evidências sugerem que a melhora dos níveis de elasticidade da pele está relacionada à integridade e funcionalidade das fibras elásticas e colagênicas; recente estudo propõe a correlação da capacidade de distensão cutânea com as medidas cutométricas de elasticidade (LEITE E SILVA et al., 2009).

\section{ATIVOS COSMÉTICOS E PREVENÇÃo} DE ESTRIAS

Hidratantes preservam o manto hidrolipídico da pele (LEONARDI;
GASPAR; CAMPOS， 2002; KEDE; SABATOVICH, 2009). A dissolução deste manto influencia nas condições de hidratação e predispõe a pele ao ressecamento e descamação (GFATTER; HACKL; BRAUN, 1997). Uma fórmula hidratante eficaz deve apresentar componentes oclusivos, umectantes e emolientes (DRAELOS, 2009).

Pesquisas avaliando fórmulas e ativos hidratantes divergem em seus resultados. Apesar de grande parte das gestantes fazer uso de um ou mais cremes/loções para prevenção de SG, não foi encontrada correlação entre o uso de creme e a profilaxia das SG por Osman et al., (2007). Contudo, autores sugerem que a aplicação preventiva de cremes de massagem a base de óleo ou água parece diminuir a incidência de SG (CROCCO; MANTOVANI; VOLPINI, 2012). A aplicação de cremes acrescida de massagem é adjuvante neste processo (GUIRRO e GUIRRO, 2004) por aumentar a micro-circulação local e melhorar a absorção dos ativos (FERNANDES, 2012).

Teste realizado a partir do creme Alphastria® composto de ácido hilalurônico, alantoína, vitamina A, vitamina E, e dexpanthenol (DE BUMAN; WALTHER; DE WECK, 1986) mostrou melhora na prevenção de SG. Ensaios com creme contendo extrato de Centellaasiatica, vitamina $\mathrm{E}$, hidrolisados de colágeno-elastina (Trofolastin®,Novartis Barcelona, Espanha)(YOUNG; JEWELL, 1996) e fórmula contendo ácido lático 
(ADDO et al., 2012), ambos com acréscimo de massagem também apresentaram melhora quanto à incidência de SG (WIERRANI et $a l ., 1992)$.

Em contraste, ativos como a manteiga de cacau (OSMAN et al., 2008; BUNCHANAN; FLETCHER; REID (2010) e óleo de oliva não se mostraram eficazes no tratamento preventivo de SG (TAAVONI et al., 2011; SOLTANIPOOR et al, 2012). Todavia, estudo antigo realizado em Londres por Davy, (1972) resultou em baixa incidência de SG nas usuárias de óleo de oliva.

\section{CONCLUSÃO}

Diante o exposto, pode-se perceber que as estrias são uma condição inestética que atingem grande parte das mulheres grávidas. A maioria das gestantes não recebe

\section{REFERENCIAS.}

ADDOR, Flávia Alvim Sant'Anna et al. Avaliação clínica de uma formulação de uso tópico como auxiliar na prevenção de estrias na gestação. Surgical\&CosmeticDermatology, v. 4, n. 4, p. 304-308, 2012.

ALVES, Gilvan; AZULAY, Luna; COSTA, Adilson. Dermatologia e gravidez. 1 . ed. Rio de Janeiro: Elsevier, 2009.

AMERICAN COLLEGE OF OBSTETRICIANS AND

GYNECOLOGISTS et al. Exercise during pregnancy and the postpartum period. Clinicalobstetricsandgynecology, v. 46, n. 2, p. 496, 2003. orientações quanto aos cuidados com a pele durante a gestação. Fatores de risco como o peso da gestante antes da gravidez, histórico familiar de estrias, peso do feto, pratica irregular de atividade física e alimentação inadequada podem deixar a mulher mais predisposta ao desenvolvimento de estrias gravídicas. A orientação sobre quais os ativos são mais eficazes e como estes devem ser utilizados levada em consideração pelo farmacêutico. A associação de mais de um ativo nas fórmulas cosméticas, sugeriu ser mais eficaz na profilaxia das estrias gravídicas. Sendo assim, o farmacêutico deve assumir seu papel quanto à orientação das gestantes. Sempre respeitando a ordem de atenção farmacêutica e aos detalhes. A intervenção deve ser realizada quando necessária, ou então encaminhar o paciente pra outros profissionais.

ATWAL, G. S. S. et al. Striaegravidarum in primiparae. British Journal of Dermatology, v. 155, n. 5, p. 965-969, 2006.

Barel AO, Paye M, Maibach HI. Cosmetic Science and Technology.Informa Healthcare. 3:1-887, 2009.

BORGES, Fábio dos Santos. Modalidades terapêuticas nas disfunções estéticas. 2 . ed. ver. E ampl. São Paulo: Phorte, 2010.

BUCHANAN, Keisha; FLETCHER, Horace M.; REID, Marvin.Prevention of striaegravidarum with cocoa butter cream. InternationalJournalofGynecology \&Obstetrics, v. 108, n. 1, p. 65-68, 2010 
CARVALHAES, Maria Antonieta de Barros Leite et al. Physical activity in pregnant women receiving care in primary health care units. Revista de saude publica, v. 47, n. 5, p. $958-967,2013$

CHANG, Anne Lynn S.; AGREDANO, Yolanda Z.; KIMBALL, Alexa Boer. Risk factors associated with striaegravidarum. Journal of the American Academy of Dermatology, v. 51, n. 6, p. 881-885, 2004.

CORMACK, David H. Fundamentos de histologia. 2. ed. Rio de Janeiro: Guanabara Koogan, 2008.

COSTA, Adilson; ALVES, G.; AZULAY, L. Dermatologia e gravidez. 2009.

COUTINHO, Gizelli Santos Lourenço et al. Prescrição de produtos dermocosméticos durante a gravidez. Ciência \& Saúde, v. 5, n. 1, p. 16-25, 2012.

CROCCO, Elisete Isabel; MANTOVANI, PatriziaAltomani; VOLPINI, Beatrice MussioFornazier. Em busca dos tratamentos para Striae Rubra e Striae Alba: o desafio do dermatologista. Surgical\&CosmeticDermato logy, v. 4, n. 4, p. 332-337, 2012.

DAMAZIO, Marlene Gabriel; GOMES, Rosaline Kelly. Cosmetologia: descomplicando os princípio ativos.4. ed. rev. São Paulo: LivrariaMédicaPaulista, 2013.

DAVEY, C. M. H. Factors associated with the occurrence of striaegravidarum. BJOG: An International Journal of Obstetrics \&Gynaecology, v. 79, n. 12, p. 1113-1114, 1972.

DE BUMAN, M.; WALTHER, M.; DE WECK, R. [Effectiveness of Alphastria cream in the prevention of pregnancy stretch marks (striaedistensae).Results of a double-blind study]. GynakologischeRundschau, v. 27, n. 2, p. 79-84, 1986.

DRAELOS, Zoe Diana. Cosmecêuticos. 2. ed. Rio de Janeiro: Elsevier, 2009.
DURMAZLAR, SeldaPelinKartal; ESKIOGLU, F. Striaegravidarum: associatedfactors in Turkishprimiparae. $\mathbf{J}$ TurkAcadDermatol, v. 3, n. 4, p. 1-5, 2009

FERNANDES, ADRIANA ISABEL PALHARES, Cuidados dermocosméticos para uma pele saudável: Aconselhamento farmacêutico nos casos mais comuns, Mestrado Integrado em Ciências Farmacêuticas - Universidade do Algarve 2012.

GARTNER, Leslie P.; HIATT, James L. Tratado de histologia em cores. 3. ed. Rio de Janeiro: Elsevier, 2007.

GFATTER, R.; HACKL, P.; BRAUN, F. Effects of soap and detergents on skin surface $\mathrm{pH}$, stratum corneum hydration and fat content in infants.Dermatology, v. 195, n. 3, p. 258-262, 1997.

GHASEMI, A. et al. Striaegravidarum: associated factors. Journal of the European Academy of Dermatology and Venereology, v. 21, n. 6, p. 743-746, 2007.

GUIRRO, Eliane C. De O.; GUIRRO, Roberto de J. Fisioterapia DermatoFuncional: fundamentos, recursos, patologias. 3. ed. São Paulo: Manole, 2004.

LEONARDI, Gislaine Ricci; GASPAR, Lorena Rigo; CAMPOS, PMBGM. Estudo da variação do $\mathrm{pH}$ da pele humana exposta à formulação cosmética acrescida ou não das vitaminas $\mathrm{A}, \mathrm{E}$ ou de ceramida, por metodologia não invasiva. AnBrasDermatol, v. 77, n. 5, p. 563-569, 2002.

JUNQUEIRA, L.C. \& CARNEIRO, J. Histologia Básica. 9. ed. Rio de Janeiro: Guanabara Koogan,1999.

KEDE, MARIA P.V.; SABATOVICH, O. Dermatologia estética. 2. ed. rev. e ampl. São Paulo: Atheneu, 2009.

LEITE E SILVA, Vânia Rodrigues et al. Hydrating effects of moisturizer active compounds incorporated into hydrogels: in vivo assessment and comparison between 
devices. Journalofcosmeticdermatology, v. 8, n. 1, p. 32-39, 2009

NASCIMENTO, S. L. et al. The effect of an antenatal physical exercise programme on maternal/perinatal outcomes and quality of life in overweight and obese pregnant women: a randomised clinical trial. BJOG: An International Journal of Obstetrics \&Gynaecology, v. 118, n. 12, p. 1455-1463, 2011.

NASCIMENTO, Simony Lira do et al. Physical exercise, weight gain, and perinatal outcomes in overweight and obese pregnant women: a systematic review of clinical trials. Cadernos de Saúde Pública, v. 27, n. 3, p. 407-416, 2011.

NUSSBAUM, Rachel; BENEDETTO, aspectos Anthony V. cosméticos de gravidez. Clínicas em dermatologia , 24 v., n. 2, p. 133-141, 2006.

MAIA, Marcus et al. Estrias de distensão na gravidez: fatores de risco em primíparas. Anais Brasileiros de

Dermatologia, v. 84, n. 6, p. 599-605, 2009.

Ministério da Saúde. Conselho Nacional de Saúde. Aprova a Política

Nacional de Assistência Farmacêutica. Resolução CNS No 338, de 06 de maio de 2004a.

MARIN, N. et al. Assistência Farmacêutica para Gerentes Municipais. Rio de Janeiro: OPAS/OMS, 2003.

MELCHIORS, Ana Carolina. Efeitos da atenção farmacêutica nos resultados em saúde dos pacientes. 2013.

MOTTOLA, Michelle F. Exercise prescription for overweight and obese women: pregnancy and postpartum. Obstetrics and gynecology clinics of North America, v. 36, n. 2, p. 301316, 2009

OSMAN, H. et al. Cocoa butter lotion for prevention of striaegravidarum: a double-blind, randomised and placebo-controlled trial*. BJOG: An

International Journal of Obstetrics

\&Gynaecology, v. 115, n. 9, p. 1138-1142, 2008.

OSMAN, Hibah et al. Risk factors for the development of striaegravidarum.American journal of obstetrics and gynecology, $v$. 196, n. 1, p. 62.e1-62. e5, 2007.

PIRES, Cezar Mario; SITTART, José A. de Souza. Dermatologia na prática médica. São Paulo: Rocca, 2007.

Ponte MG. (2012) Recursos

fisioterapêuticos utilizados no tratamento das estrias: uma revisão de literatura Pósgraduação em Fisioterapia Dermato-

Funcional - Faculdade Ávila.1-12

SALTER, Sharon A. et al. Striae and pelvic relaxation: two disorders of connective tissue with a strong association. JournalofInvestigativeDermato logy, v. 126, n. 8, p. 1745-1748, 2006.

SCHEEREN, Carine. Motivação do médico dermatologista em optar, no momento da prescrição, pelo medicamneto manipulado ou industrializado. 2009.

SOLTANIPOOR, F. et al. The effect of olive oil on prevention of striaegravidarum: A randomized controlled clinical trial. Complementary therapies in medicine, v. 20, n. 5, p. 263-266, 2012.

TAAVONI, Simin et al. Effects of olive oil on striaegravidarum in the second trimester of pregnancy. Complementarytherapies in clinicalpractice, v. 17, n. 3, p. 167-169, 2011. TEIXEIRA, Vera et al. Dermatoses Específicas da Gravidez. Acta Médica Portuguesa, v. 26, n. 5, p. 593-600, 2013.

THOMAS, R. G. R.; LISTON, W. A. Clinical associations of striaegravidarum. Journal of Obstetrics \& Gynecology, v. 24, n. 3, p. 270271, 2004.

URASAKI, Maristela Belletti Mutt. Cuidados com a pele adotados por gestantes assistidas 
em serviços públicos de saúde. Acta Paul

Enferm, v. 24, n. 1, p. 67-73, 2011.

WATSON, R. E. B. et al. Fibrillinmicrofibrils are reduced in skin exhibiting

striaedistensae. British

JournalofDermatology, v. 138, n. 6, p. 931-

937, 1998.

WIERRANI,F.; KOSAK, W.; SCROMM,W.; GRUNBERGER,W. Attempt of preventive treatament of striaegravidum using preventive massage ointment administration. Wien-

Klinwochenschr,104:42-4,1992.

YOSIPOVITCH, Gil; DEVORE, Amy;

DAWN, Aerlyn. Obesity and the skin: skin physiology and skin manifestations of obesity. Journal of the American Academy of Dermatology, v. 56, n. 6, p. 901-916, 2007.

YOUNG, G. L.; JEWELL, David.Creams for preventing stretch marks in pregnancy. Cochrane

DatabaseofSystematicReviews, v. 1, 1996. 